\title{
An analytical modeling method for calculating the maximum cathode current deliverable by a circular cathode under atmospheric exposure
}

\author{
Z. Y. Chen \& R. G. Kelly \\ Center for Electrochemical Science and Engineering, \\ Department of Materials Science and Engineering, \\ University of Virginia, Charlottesville, USA
}

\begin{abstract}
An analytical method for evaluating the stability of pitting corrosion of corrosion-resistant alloys under thin-layer (or atmospheric) conditions is presented. The method uses input data that are either thermodynamic in nature or easily obtained experimentally. The maximum cathode current available (referred to as the cathode capacity) depends on the cathode geometry, temperature, relative humidity, deposition density of salt (i.e., mass of salt per unit area of cathode), and interfacial electrochemical kinetics. The anode demand depends on the pit geometry and the pit stability product. By coupling these two approaches, the stability of a pit can be determined for a given environmental scenario. The method has been applied to the atmospheric pitting corrosion of Type 316L stainless steel, leading to a quantitative description of limiting stable pit sizes.
\end{abstract}

Keywords: galvanic corrosion, relative humidity, $\mathrm{NaCl}$ particles, localized atmospheric corrosion, stainless steel, pitting.

\section{Introduction}

Corrosion resistant materials exposed to marine atmospheric conditions can suffer from localized corrosion (pitting, crevice corrosion, stress-corrosion cracking). The stability of such a localized corrosion site requires that the site (anode) must dissolve at a sufficiently high rate to maintain its critical chemistry [1] and a wetted surrounding area (cathode) must provide a matching cathodic 
current. The objective of this study was to computationally characterize the stability of such a local corrosion site and explore the effects of physiochemical parameters and electrochemistry of this stability. Specifically, this work considers the stability of a pit on Type 316L stainless steel exposed to atmospheric conditions after the deposition of a known amount of sodium chloride.

\subsection{Background}

The conservation of charge requires that the sum of the cathodic reaction rates be equal to the sum of the anodic reaction rates for a corrosion system under open circuit conditions. A stable localized corrosion system (depassivated anode, dominant external cathode) requires that the two regions be compatible in terms of the current demanded by the anode and the current that could be supplied by the cathode.

In localized corrosion, it is generally accepted that the stability of the anode site requires that its dissolution rate be sufficient to maintain an aggressive solution against the diluting effects of diffusion. The aggressiveness required is a function of the alloy composition, bulk environment, and temperature. Galvele [2-4] was the first to address this issue analytically, and considered the diffusion and migration relevant to the pit, assuming that there was sufficient cathodic reaction to maintain electroneutrality. Galvele demonstrated that for a onedimensional pit with an active base and passive walls, the stability parameter could be put in terms of the dissolution rate at the base of the pit, $i$, and the depth of the pit, $x$, in the form of a pit stability product, $i \cdot x$. For a hemispherical pit, it has been shown that the stability product takes the form $I / r$ where $I$ is the anodic current from the pit, and $r$ is its radius [5,6]. Several researchers have experimentally determined the so-called pit stability product for different materials, and values are generally close to $10^{-2} \mathrm{~A} / \mathrm{cm}$ for stainless steel $[5,7,8]$.

Although the pit stability product has been successfully used to assess the minimum current required for a pit to be stable, little work has been done considering what controls the current that a cathode can supply, particularly under atmospheric exposure conditions.

For most localized corrosion systems, the vast majority of the cathodic current is supplied by the surface outside the localized corrosion site. For atmospheric exposures, only thin layers of electrolyte will be present. These layers represent a substantial ohmic resistance between the localized corrosion site and more remote areas on the cathode. This resulting ohmic drop distributed along the cathode will limit the total cathodic current that it can provide in support of the localized corrosion site.

Recently, Cui et al. [9] and Kelly et al. [10] presented an analytical solution for a one-dimensional cathode which allowed a solution for the cathode current capacity at a constant temperature as a function of interfacial kinetics, $\mathrm{RH}$, deposition density, and the deliquescence behavior of the salt deposited. For stability considerations, knowledge of only the total net current from the cathode, $I_{n e t}$, is required; its distribution along the cathode is unimportant. Thus, an 
equivalent cathode can be constructed which provides the same current as the actual cathode, but has a configuration that allows a straightforward calculation of $I_{\text {net }}$. They validated the approach by comparisons of analytic calculations to the results of finite element analysis methods. In addition, they showed that a bounding solution can be found, termed $I_{n e t, \max }$, which describes the maximum current available from a given cathode irrespective of its size. This maximum, referred to as the cathode current capacity, results from the fact that once an area of the cathode is sufficiently far from the anode that the potential drop leads to an interfacial potential of the open circuit corrosion potential of the cathode, it cannot provide any net cathodic current to the anode as it is all being used locally to support the passive dissolution.

Extending the approach of Cui et al. [9] and Kelly et al. [10] to a circular cathode is required to address the less restrictive mass transport conditions of relevance to a pit surrounded by a wetted area. Pitting is a very common form of corrosion damage for stainless steels exposed to marine atmospheres.

\section{Computational approach}

We follow the approach of Kelly et al. [10] in defining an equivalent cathode as shown in Figure 1. Figure 1 shows a one-dimensional cathode, but the tenets are applicable for two-dimensional cathodes as well. As mentioned above, the issue of localized corrosion stability requires knowledge of the total net cathodic current, not its distribution along the cathode. Thus, we can more easily calculate the $I_{\text {net }}$ by defining an equivalent current density, $i_{e q}$, and an equivalent length (in 1D), $L_{e q}$, that results in the same $I_{n e t}$ as the actual cathode.

$$
I_{n e t}=i_{e q} \cdot L_{e q} \cdot W
$$

The equivalent current density is defined by:

$$
i_{e q}=\frac{\int_{E_{L}}^{E_{r p}}\left(i_{c}-i_{p}\right) \cdot d E}{E_{L}-E_{r p}}
$$

where $\left(i_{c}-i_{p}\right)$ is the cathodic current less the passive current at a given potential (i.e., the electrochemical kinetics of the cathode), $E_{r p}$ is the repassivation potential which is assumed to be that at the mouth of the pit, $E_{L}$ is the potential at the position on the cathode furthest from the pit, and $\mathrm{W}$ is the width of the cathode.

If we consider only ohmic drop along the cathode, then the difference in potential between the anode and the farthest point on the cathode would be related to the current and the nominal resistance: 


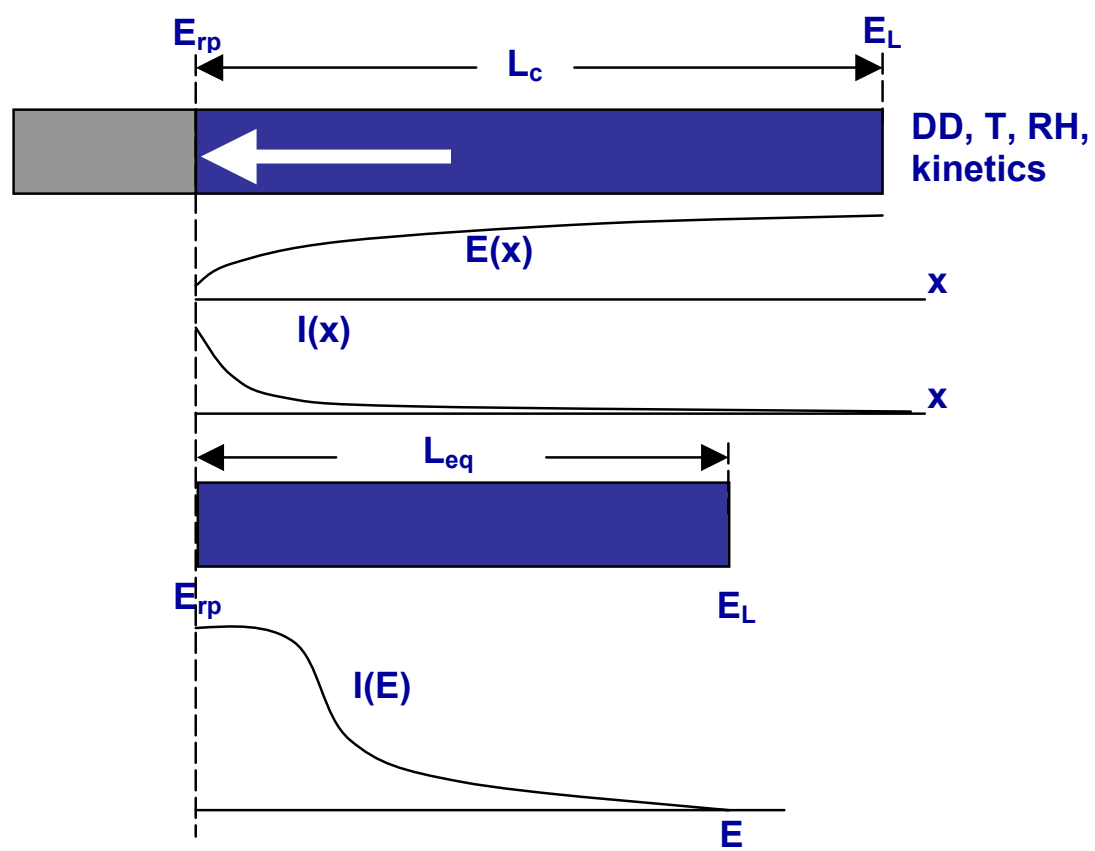

Figure 1: Schematic showing the connection between a real 1D cathode and an equivalent $1 \mathrm{D}$ cathode.

$$
\Delta E=I \cdot R=\frac{\int_{E_{L}}^{E_{r p}}\left(i_{c}-i_{p}\right) \cdot d E}{E_{L}-E_{r p}} \cdot \frac{L_{e q}^{2}}{2 \cdot \kappa \cdot W L}
$$

Combing eqns (1) through (3) results in the expression for $I_{n e t}$ :

$$
I_{n e t}=W \cdot \sqrt{2 \cdot \kappa \cdot W L \cdot \int_{E_{L}}^{E_{r p}}\left(i_{c}-i_{p}\right) \cdot d E}
$$

where $\kappa$ is the conductivity of the electrolyte layer and WL is the thickness of the electrolyte layer.

The accuracy of eqn (4) has been validated by independent FEM calculations of the current distributions under the same conditions [10]. Replacing $E_{L}$ with the open circuit potential of the cathode, $E_{\text {corr }}$, leads to an expression for the maximum net cathodic current that a cathode can deliver under the conditions considered. 
Extending this approach to a circular geometry requires a new definition of the anode and cathode dimensions as shown in Figure 2. The central anode (the pit) has a radius $r_{a}$ and the equivalent cathode has a radius $r_{e q}$. The $I_{n e t}$ is defined in an identical way to the $1 \mathrm{D}$ case. Consideration of ohmic drop results in an expression for $I_{\text {net }}$ :

$$
\ln I_{n e t}=\frac{4 \pi \cdot \kappa \cdot W L \cdot\left(E_{L}-E_{r p}\right)}{I_{n e t}}+\ln \left(\frac{\pi \cdot e \cdot r_{a}^{2} \cdot \int_{E_{L}}^{E_{p}}\left(i_{c}-i_{p}\right) \cdot d E}{E_{L}-E_{r p}}\right)
$$

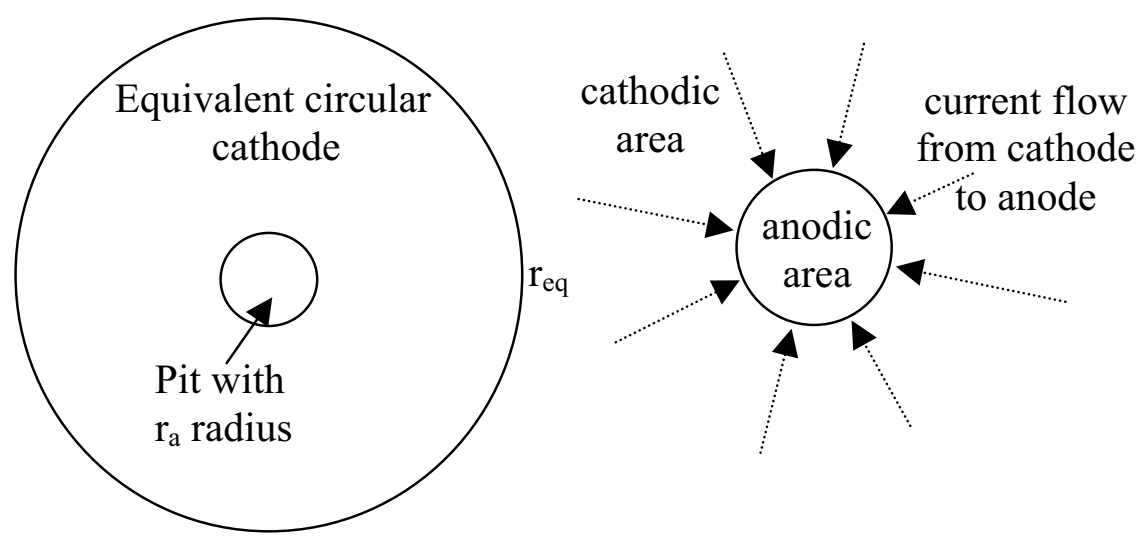

Figure 2: Schematic showing the circular cathode around a pit and the radial current distribution.

Again, replacement of $E_{L}$ with $E_{\text {corr }}$ leads to an expression for $I_{\text {net, } \max }$.

The $W L$ and $\kappa$ are difficult to control or measure in either the laboratory or the field. However, under equilibrium conditions, their values can be calculated knowing the relative humidity $(R H)$, the amount of salt deposited per unit area (hereafter referred to as the deposition density, $D D$ ), and quantitative information about the deliquescence behavior of the deposited salt [11]. Deliquescence data are available for a wide range of salts and mixtures of salts as a function of temperature.

\section{Results}

Figure 3 shows a comparison of the results of the analytical expression (eqn (5)) with the results of FEM analyses of the same conditions. In the case of the FEM analyses, the current distributions were determined, then integrated over the length of the cathode. The agreement is excellent over more than two orders of magnitude of current.

One means for using eqn (5) is to assess the effect of $R H$ and $D D$ on the $I_{\text {net,max }}$. Figure 4 shows an example for Type 316L stainless steel. The cathode 
kinetics were taken from the literature, with the diffusion limited current density adjusted to be equal to the water layer thickness. For $W L<200 \mu \mathrm{m}$, this has been demonstrated experimentally to be the case [12].

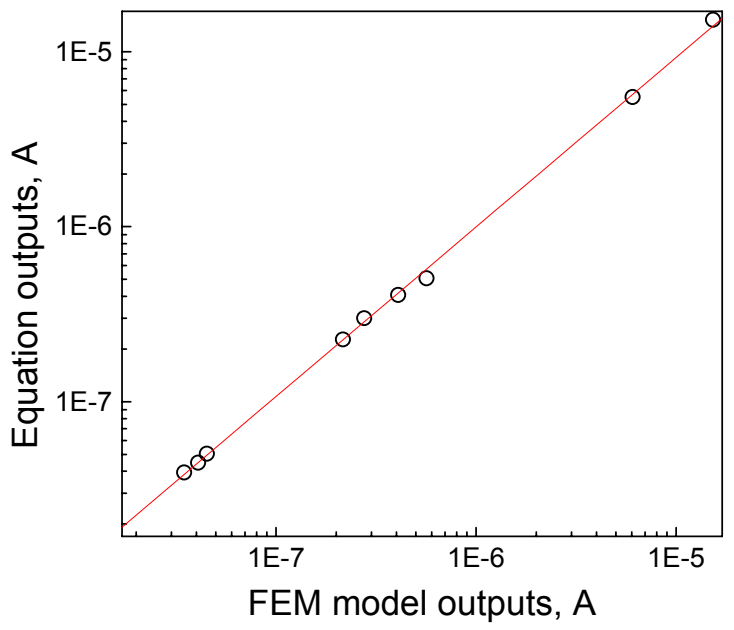

Figure 3: Comparison of the net cathodic current results obtained from finite element model calculations and from eqn (5).

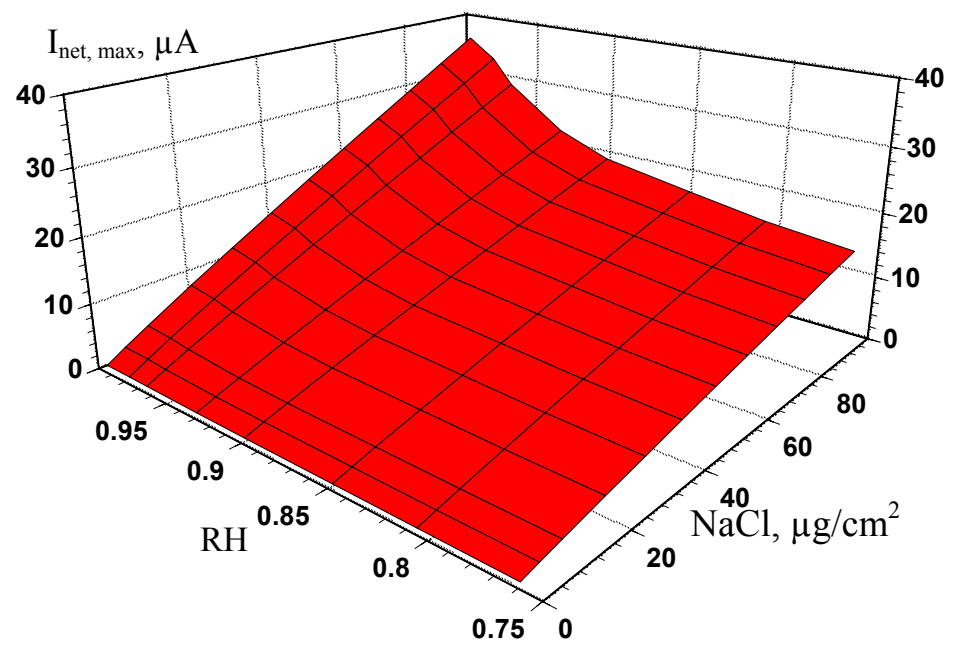

Figure 4: The effect of relative humidity and amount of deposited $\mathrm{NaCl}$ particles on the cathode capacity of SS 316L under atmospheric exposure. The cathode is circular around a pit with radius of $10 \mu \mathrm{m}$. Temperature is $25^{\circ} \mathrm{C}$. 


\section{Discussion}

An important application of the $I_{\text {net } \max }$ is its coupling to the pit stability product. Pits in stainless steels often grow on the edge of stability even under potentiostatic conditions [5]. In atmospheric exposures, the limitations on cathode current available can become important in determining the stability of the localized corrosion system.

In considering such an analysis, the bounding nature of $I_{\text {net.max }}$ must be justified. As constituted, the $I_{\text {net, max }}$ expression assumes that the chemistry of the surface solution is constant, there are no inert particulate on the surface, and the solution perfectly wets the a flat surface. Each of these represents a practical upper bound. In reality, the solution chemistry will evolve with time, typically increasing in $\mathrm{pH}$ due to the production of hydroxyl on the cathode surface via reduction of oxygen and/or water. Higher $\mathrm{pH}$ solutions lead to slower cathodic kinetics, thus leading to a decrease in the term within the integral of eqn (5), and lower $I_{\text {net }}$. In service, inert particulate are usually present on surfaces. These particulate lower the effective conductivity of the surface, which leads directly to a decrease in the $I_{\text {net }}$. Finally, surface tension considerations as well as surface curvature, result in physical limitations on the cathode size which would lower $I_{\text {net. }}$.

Figure 5 illustrates the coupling of the $I_{n e t, \max }$ expression and the pit stability product for Type 316L stainless steel exposed to a marine environment. It considers two deposition densities $\left(10\right.$ and $\left.100 \mu \mathrm{g} / \mathrm{cm}^{2}\right)$ and two RH (85 and $98 \%)$ at $25^{\circ} \mathrm{C}$. A pit stability product of $10^{-2} \mathrm{~A} / \mathrm{cm}$ is also assumed $[5,7,8]$. As is obvious from the definition of the pit stability product, as the pit grows, the minimum current it requires increases linearly with $r_{a}$. As noted in Figure 5, $I_{n e t, \max }$ also increases with increasing $r_{a}$, but at a less than linear rate. The intersection of the $I_{n e t, \max }$ and $I_{L C}$ curves represents the maximum pit size that the cathode can support. A series of critical pit radii are shown for the different $\mathrm{DD} / \mathrm{RH}$ combinations, ranging from $1.5 \mu \mathrm{m}$ (for $85 \% \mathrm{RH}, 10 \mu \mathrm{g} / \mathrm{cm}^{2}$ ) to 39.1 $\mu \mathrm{m}$ (for $98 \% \mathrm{RH}, 100 \mu \mathrm{g} / \mathrm{cm}^{2}$ ).

Pits smaller than the critical pit radius can grow, whereas once the critical pit radius is reached, the pit must either repassivate, or have only a portion of its surface remain active. The latter choice is an example of a type of stifling of localized corrosion.

Note that this type of analysis does not guarantee that pits will reach the maximum size. As noted above, the $I_{\text {net, } \max }$ equation represents an upper bound. If conditions are such that the actual $I_{n e t}$ is less than $I_{n e t, \max }$, then the maximum supportable pit size will be smaller.

\section{Conclusions}

A bounding analytical solution for the maximum current that a circular cathode can deliver $\left(I_{\text {net, max }}\right)$ to a pit exposed to a marine atmosphere has been derived and validated numerically. The solution shows that there is a linear dependence of $I_{n e t, \max }$ on deposition density. Coupling of the analytical expression for the $I_{n e t, \max }$ 
for the cathode with the Galvele pit stability product sets a defined limit on the rate at which a semicircular pit can grow as well as a maximum size for a given exposure scenario ( $\mathrm{T}$, deposition density, RH, cathode interfacial kinetics, anode critical pit stability product).

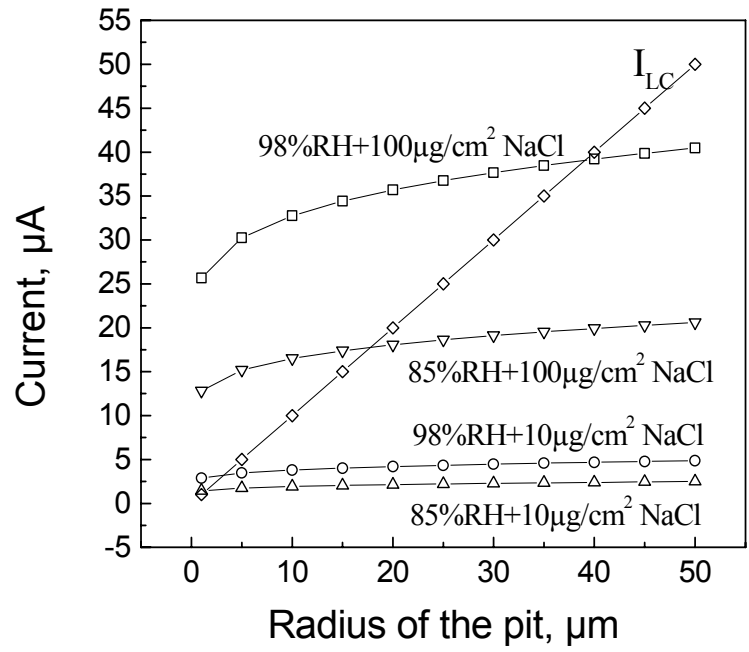

Figure 5: The effect of the radius of the pit on the circular cathode capacity, $I_{n e t, \max }$, and anodic current demand, $I_{L C}$.

\section{Acknowledgements}

Support by the Office of Science and Technology and International of the U.S. Department of Energy (DOE), Office of Civilian Radioactive Waste Management is gratefully acknowledged. The work was performed under the Corrosion and Materials Performance Cooperative, DOE Cooperative Agreement Number: DE-FC28-04RW12252.

\section{References}

[1] Galvele, J. R., Tafel's law in pitting corrosion and crevice corrosion susceptibility. Corros. Sci., 47(12), pp. 3053-3067, 2005.

[2] Galvele, J. R., Transport processes and the mechanism of pitting of metals. J. Electrochem. Soc. 123(4), pp. 464-474, 1976.

[3] Galvele, J. R., Transport processes in passivity breakdown-II. Full hydrolysis of the metal ions. Corros. Sci. 21(8), pp. 551-579, 1981.

[4] Gravano S. M. \& Galvele, J. R., Transport processes in passivity breakdown-III. Full hydrolysis plus ion migration plus buffers. Corros. Sci., 24(6), pp. 517-534, 1984. 
[5] Williams, D.E., Stewart, J. \& Balwill, P. H., "Nucleation, growth, and stability of micropits in stainless steels," in Critical Factors in Localized Corrosion, Frankel, G. S. and Newman, R. C., Editors, PV 92-9, The Electrochemical Society Proceedings Series, Pennington, NJ, pp. 36-64, 1992.

[6] Pride, S. T., Scully, J. R. \& Hudson, J. L., Metastable pitting of aluminum and criteria for the transition to stable pit growth. J. Electrochem. Soc., 141(11), pp. 3028-3040, 1994.

[7] Burstein, G. T., Pistorius, P. C. \& Mattin, S. P., The nucleation and growth of corrosion pits on stainless steel. Corros. Sci., 35(1-4), pp. 57 62, 1993.

[8] Moayed, M. H., \& Newman, R. C., The relationship between pit chemistry and pit geometry near the critical pitting temperature. $J$. Electrochem. Soc., 153(8), pp. B330-B335, 2006.

[9] Cui, F., Preseul-Moreno, F. J. \& Kelly, R. G., Computational modeling of cathodic limitations on localized corrosion of wetted SS 316L at room temperature. Corros. Sci., 47(12), pp. 2987-3005, 2005.

[10] Kelly, R. G., Cui, F. \& Preseul-Moreno, F. J., Computational modeling of the stability of crevice corrosion of wetted SS316L. ECS Trans., 1(16) pp. 17-36 (2006).

[11] Chen, Z. Y., Cui, F. \& Kelly, R. G., "An Analytical Modeling Method for Calculating the Current Delivery Capacity of a Thin-Film Cathode and the Stability of Localized Corrosion under Atmospheric Environments," accepted by ECS Trans, November, 2006.

[12] Nishikata, A., Ichihara, V., Hayashi, V. \& Tsuru, T., Influence of electrolyte layer thickness and $\mathrm{pH}$ on the initial stage of the atmospheric corrosion of iron. J. Electrochem. Soc., 144(4), pp. 1244-1252, 1997. 